\title{
Inégalités et cancers : ce que recouvre une réalité clinique
}

\author{
Cancer and inequality: what is hidden by the clinical reality?
}

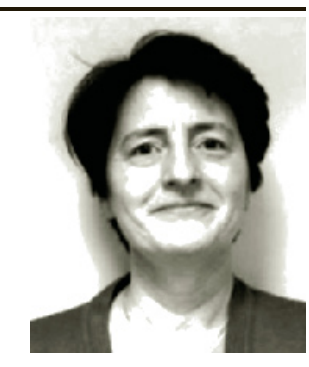

\section{N. Pélicier}

C) Springer-Verlag France 2010

La SFPO a organisé son $27^{\mathrm{e}}$ Congrès autour de la notion d'inégalités face aux cancers, notion richement documentée en santé publique. Mais qu'en est-il du corrélat psychique en termes de résonance et de psychopathologie?

De quelle inégalité parle-t-on, dans un registre pluridisciplinaire qui est celui de nos pratiques cliniques particulièrement en psycho-oncologie ? Les regards croisés des épidémiologistes, des cancérologues soucieux d'afficher la disparité des conditions, des origines, voire des causalités facteurs d'inégalités, correspondent-ils à ceux des patients ou des familles ? Pour eux, l'inégalité des destins alimente un monde de représentations où l'injustice côtoie l'insécurité.

Ce congrès et ce numéro de Psycho-Oncologie se veulent une occasion d'alerter les acteurs du soin en cancérologie, mais aussi les responsables en santé publique sur les enjeux psychiques multiples de la question du cancer : enjeux bouleversant les campagnes de dépistage, l'approche diagnostique, le vécu des traitements et l'après-maladie. Trop peu étudiés et considérés ou, au contraire, banalisés comme une évidence, ces enjeux se manifestent en soulignant, amplifiant, voire déclenchant des sources profondes d'inégalités du parcours de soins.

L'accès à l'information, mais aussi aux soins, les conséquences à long terme de la maladie, qui nous sont restitués, nous invitent à une relecture qui déplace la seule vision de l'inégalité vers celle des diversités et des différences.

Le travail des psychiatres et des psychologues cliniciens au sein des services ou des réseaux de cancérologie a permis de souligner davantage les phénomènes psychopathologiques réactionnels aux cancers, mais aussi de réhabiliter l'histoire singulière du sujet. Les facteurs de risque, mais aussi les antécédents psychiques, souffrance ou vulnérabilité, éclairent la scène des "comportements en santé ", des « attitudes » déroutant proches et équipes médicales.

\section{N. Pélicier $(\bowtie)$}

Présidente de la SFPO

Psychiatre - Service du Pr S. Consoli,

Hôpital européen Georges-Pompidou (HEGP),

20, rue Leblanc, F-75908 Paris cedex 15, France

e-mail : nicole.pelicier@egp.aphp.fr
Comprendre, et ne pas seulement « repérer », prendre en soin cette vulnérabilité psychique, permettent d'élaborer de « bonnes pratiques » en lien avec des situations de perte de chance, de réticence ou de refus de soins. Les options pronostiques, qu'il s'agisse de l'évolution dans la maladie ou de la réinsertion sociale, peuvent dépendre de notre attention au statut psychique des patients. La psycho-oncologie, on le sait, interpelle aussi les caisses de résonance que constituent le vécu des proches et celui des équipes. La justesse des soins psychiques s'appuie, pour nous, sur des interventions avant tout marquées par le professionnalisme des acteurs du soin psychique.

Nous retrouvons dans cette optique, les facteurs d'inégalité comme une réalité existentielle et psychique et non un simple écart à réduire. Éviter l'illusion, l'utopie égalitaire en santé est certes difficile...

Malgré tous les efforts du soin cancérologique, les groupes sociaux, familiaux exercent leur pression et répercutent un jeu de contrastes vécus comme inégalitaires.

Disparité voisine alors avec désespoir.

Le paradoxe de l'acceptabilité ou de la recevabilité de l'information ou du soin relance encore le débat.

Face aux possibilités de diagnostic, de traitements, surgit le désir de savoir ou pas, d'en réchapper ou pas, de revenir dans le jeu social ou de ne pas vouloir être un héros ordinaire « égal de l'autre»?

Tous les aléas du subjectif viennent alors brouiller les espoirs des uns, les repères des autres.

Ainsi, la quête égalitaire recouvrant de bonnes intentions peut masquer des réalités opportunes. Les «psy » restituent dans leur pratique ce que l'inventivité subjective suscite en termes de défense face à la maladie. Défenses nécessaires qui défient l'homogénéité des " groupes à risque ».

Conservons donc l'hétérogénéité des regards pour mieux y voir.

Le contrat social est celui de moyens suffisants pour garantir un maximum de chances. La spécificité des prises en charge, la densité des échanges avec les équipes de soins visent à déjouer des situations de crise vécues jusque-là dans l'impuissance et l'incompréhension. 
Force est de constater qu'il est possible de réduire ou de contourner certaines inégalités d'ordre social. Il reste de notre responsabilité, d'appeler à la vigilance sur une diversité propre aux langages psychiques, pour ne pas risquer une lecture psychique désubjectivisée, comprise dans un protocole d'intervention, un accompagnement « acceptable par tous ».
Trouver une mesure appropriée, « personnalisée », nous tire vers une égalité pour chacun.

Nous vous invitons à prendre ici conscience de la part irréductible, précise et subtile des enjeux psychiques à propos des cancers.

L'équité du soin est alors une recherche, une tension, non une utopie. 